\title{
Candida albicans Measurement
}

National Cancer Institute

\section{Source}

National Cancer Institute. Candida albicans Measurement. NCI Thesaurus. Code C161404.

The determination of the amount of Candida albicans present in a sample. 